\title{
Colocación de autoinjerto estructural para defecto óseo tibial medial en artroplastia total de rodilla. Reporte de un caso y revisión de la literatura
}

\author{
Autologous bone graft for medial metaphyseal tibial defects in total \\ knee arthroplasty. Case report and literature review \\ Mauricio Alonso Flores Araujo, * Marlene Vanessa Salcido Reyna, ${ }^{\ddagger}$ \\ Alejandro Bejarano González, ${ }^{\ddagger}$ Nadia Estavillo Martínez, ${ }^{*}$ Iván Topete Vergara* \\ *Médico residente de Traumatología y Ortopedia; ¥Médico adscrito de Traumatología y Ortopedia. Hospital General de Mexicali, Secretaría de Salud, Baja \\ California, México.
}

Resumen

Los defectos periféricos óseos tibiales pueden ser comúnmente encontrados en pacientes con grados avanzados de artrosis que se someten a artroplastia total de rodilla. Dentro de los métodos más comúnmente utilizados para el soporte tibial en defectos óseos metafisarios se encuentra el uso de cemento, uso de implantes especializados, injerto autólogo, entre otros. Se presenta el caso de paciente sometida a artroplastia total de rodilla con defecto óseo metafisario medial, el cual es tratado con injerto autólogo de corte femoral.

Palabras clave: Artoplastia, rodilla, defecto óseo tibial, autoinjerto, Windsor.

Abstract

Metaphyseal tibial bone defects are commonly encountered during total knee arthroplasty in patients with severe arthrosis. Some of the options for treatment of this defects include the use of cement, specialized implants and autologous bone graft among others. We present the case of a patient with medial methapyseal bone defect treated with autologous femoral cutting graft.

Keywords: Arthroplasty, knee, oseous tibial defect, autologous graft, Windsor.

\section{Introducción}

Los defectos periféricos óseos no contenidos en la superficie articular tibial pueden ser encontrados en pacientes que se someten a artroplastia total de rodilla primaria o de revisión. Estos defectos deben ser corregidos al momento de la cirugía para obtener una plataforma estable sobre la cual colocar el componente tibial. ${ }^{1}$

Aunque los resultados en artroplastia total de rodilla son excelentes en el ámbito mundial, el manejo de pérdida ósea representa un reto

Correspondencia:

Mauricio Alonso Flores Araujo

E-mail: mauricio.floress@live.com

Recibido: 10-02-2021. Aceptado: 17-02-2021. significativo durante este procedimiento. El tratamiento quirúrgico para la pérdida ósea incluye el uso de cemento, injerto de hueso autólogo, megaprótesis, prótesis en bisagra y aumentos metálicos. Las ventajas del uso de hueso autólogo para los defectos tibiales en artroplastia primaria de rodilla incluyen biocompatibilidad, disponibilidad de injerto, menor tasa de infección a comparación de injerto alogénico y facilidad de uso. ${ }^{2}$

Las limitaciones potenciales del uso de hueso autólogo como injerto incluyen la posible no unión, falta de disponibilidad de hueso para injerto y reabsorción ósea. ${ }^{3}$ 
En 1991 Rand $^{4}$ propuso una clasificación que considera el porcentaje de la extensión del defecto óseo en el platillo tibial o el cóndilo femoral, estadificando según la severidad de la lesión en cuatro grados.

El índice de unión del autoinjerto en la artroplastia total de rodilla no es bien conocido debido a la escasez de estudios que han sido realizados, el número limitado de casos y la aumentada prevalencia de este tipo de defectos en cirugías de revisión a comparación de procedimientos primarios. ${ }^{2}$

Sin embargo, varias series de casos han sido publicados con resultados excelentes cuando se realiza una adecuada selección de pacientes. ${ }^{5-7}$

\section{Presentación del caso}

Se presenta caso de mujer de 62 años, dedicada al cuidado del hogar, con antecedente de presbiacusia, la cual acude a consulta externa por gonalgia bilateral, de predominio izquierdo, de siete años de evolución. A la exploración física se aprecia franca

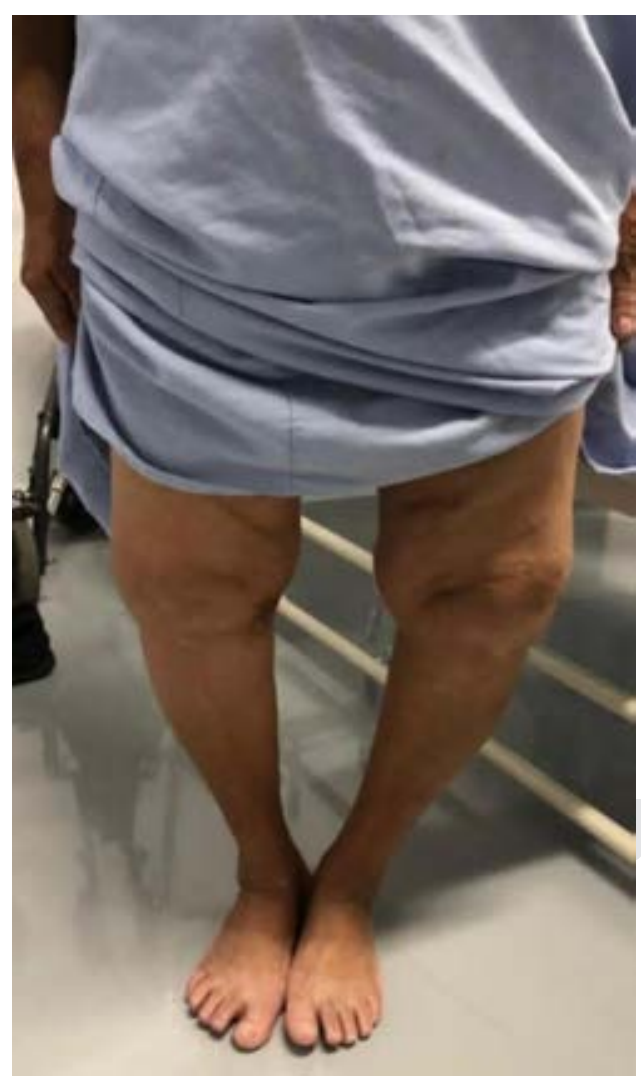

Figura 1: Genu varo bilateral prequirúrgico.

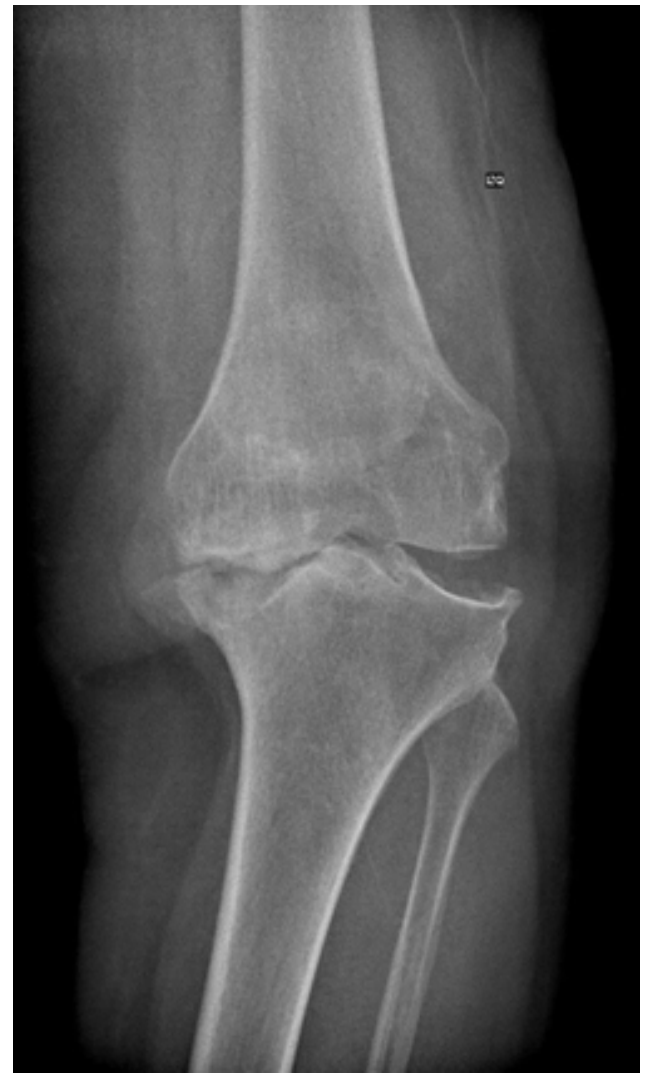

Figura 2: Radiografía anteroposterior de rodilla izquierda en bipedestación.

deformidad en varo en ambas rodillas (Figura 1), de mayor magnitud en lado izquierdo, con marcha independiente asistida con bastón, rango de movilidad activo con extensión de $-5^{\circ}$, flexión de $85^{\circ}$, Ficat y cepillos positivos, cajones negativos y bostezo medial positivo, con limitación por dolor para las actividades de la vida diaria y dolor en EVA de 8 . Se solicitan y revisan radiografías anteroposterior (Figura 2) y lateral (Figura 3) de ambas rodillas en bipedestación, encontrando gonartrosis bilateral Kellgreen \& Lawrence IV, con defecto tibial medial en rodilla izquierda, por lo cual se le propone tratamiento quirúrgico con artroplastia total de rodilla izquierda posteroestabilizada, debido a la mayor sintomatología en dicho lado.

\section{Técnica quirúrgica}

Se realiza abordaje anterior a rodilla izquierda con artrotomía transvasto, luxando patela sin eversión, hacia lateral, se retiran osteofitos femorales y ligamento cruzado anterior y posterior, y se realiza 
corte femoral distal con valgo de $7^{\circ}$, posteriormente realizando corte tibial en $0^{\circ}$. Se mide tamaño de plantilla femoral, siendo de tamaño 5 , y se realizan cortes anteriores, posteriores y chaflanes en fémur con $3^{\circ}$ de rotación externa. Se visualiza defecto tibial de $5 \mathrm{~mm}$ de profundidad (Figura 4) y se regulariza borde con sierra para hueso, obteniendo lecho plano y sangrante, adecuado para colocación de injerto. Se recupera bloque de hueso para injerto proveniente de osteotomía de corte femoral y se coloca en zona de defecto tibial realizando osteodesis con clavos Kirschner de 1.6 $\mathrm{mm}$ (Figura 5). Después se coloca nuevamente plantilla de corte tibial y se regulariza altura de injerto con sierra, procediendo a fijación definitiva con tornillos de esponjosa $4.0 \mathrm{~mm}$ de rosca com-

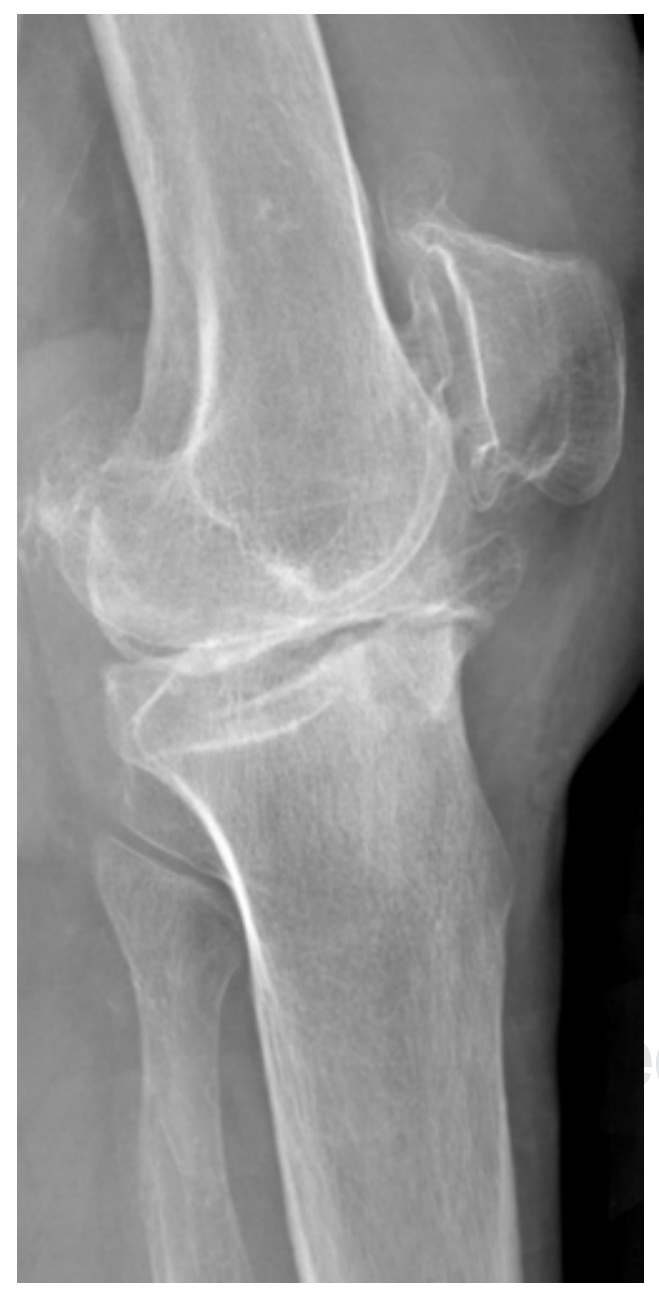

Figura 3: Radiografía lateral de rodilla izquierda en bipedestación.

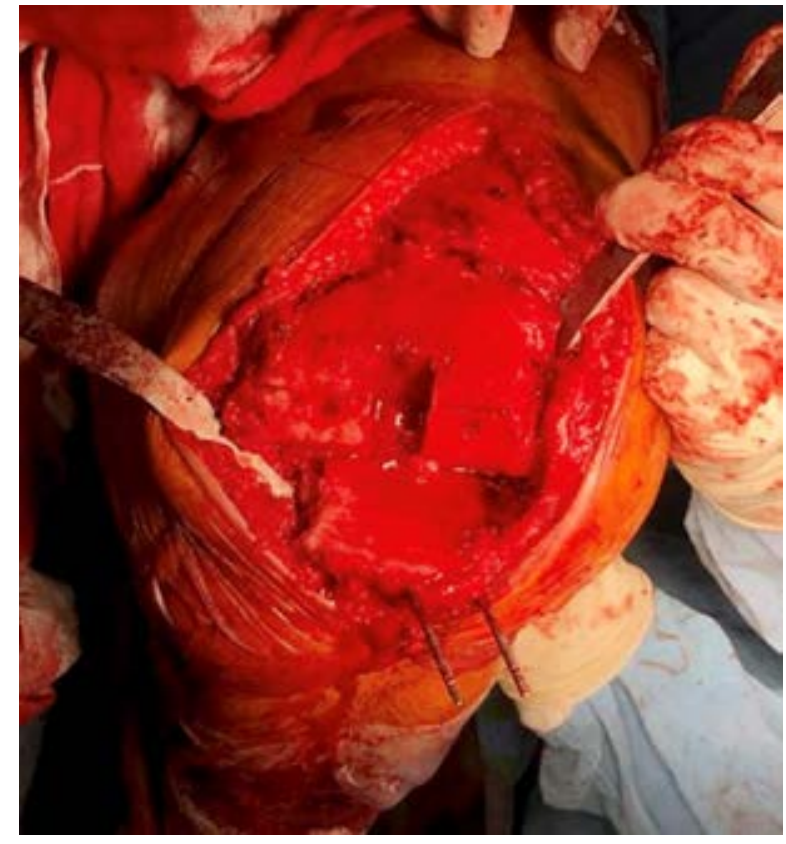

Figura 4: Platillo tibial con defecto metafisiario medial.

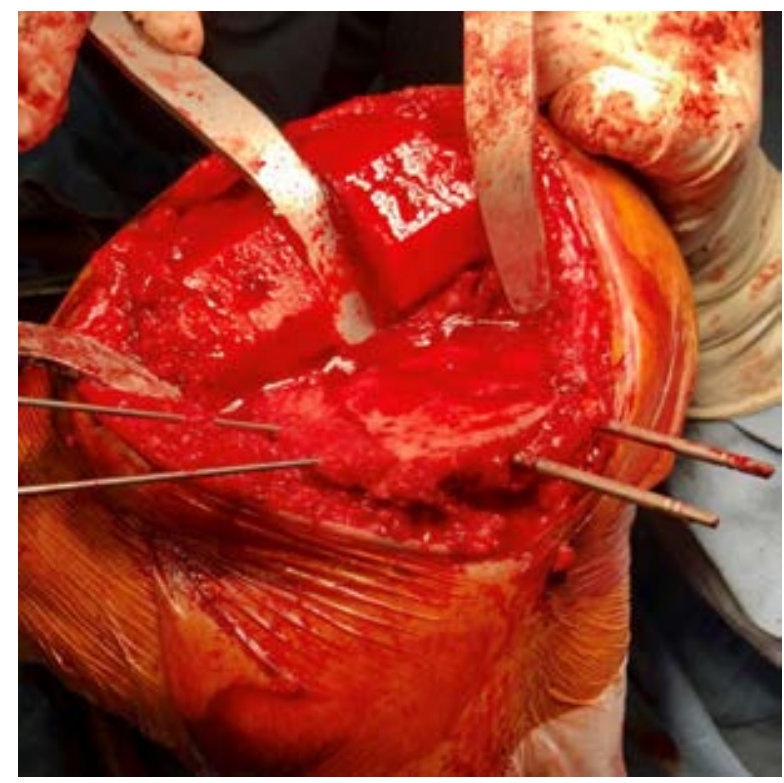

Figura 5: Platillo tibial con autoinjerto colocado, fijado provisionalmente con clavos Kirschner.

pleta con dirección de medial a lateral, cuidando no invadir espacio metafisario central para colocación de implante tibial. Se introduce espaciador de 10 $\mathrm{mm}$ en espacio tibiofemoral y se realiza balanceo de tejidos blandos con técnica de pie crusting 


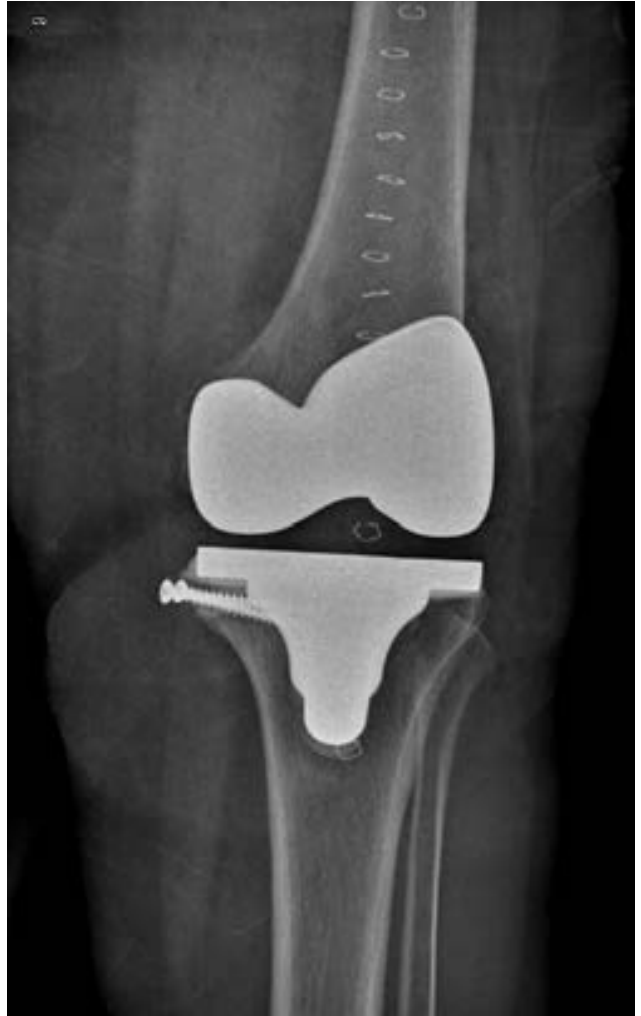

Figura 6: Radiografía anteroposterior de rodilla izquierda en postquirúrgico inmediato.

sobre estructuras mediales hasta obtener gaps simétricos en flexión y extensión. Se mide tamaño de componente tibial, siendo de tamaño 3 , y se determina rotación adecuada alineando punto medio de plantilla con la unión del tercio medial con tercio medio de tuberosidad tibial anterior, después se realiza perforación metafisaria, sin interferencia de tornillos de fijación de injerto. Se coloca plantilla de prueba y se procede a medir y realizar corte patelar para componente de tamaño 38 con medialización de componente. Se verifica adecuada movilidad y estabilidad, retiro de componentes de prueba, se coloca isquemia, irrigación de lecho quirúrgico, sellado interfase entre injerto y metáfisis tibial con cemento y se procede a colocar implantes definitivos cementados, con inserto 10 posteroestabilizado (Figuras 6 y 7). Se verifican rangos de movimiento alcanzando flexión de $130^{\circ}$ y extensión de $0^{\circ}$, con adecuado balanceo de tejidos blandos, después se cierra por planos hasta piel.

La paciente es egresada al día siguiente de procedimiento quirúrgico con indicación de mantener extre- midad pélvica izquierda en descarga hasta encontrar datos radiográficos de consolidación en injerto, y se cita a revisión a los siete, 14 y 28 días después del procedimiento (Figuras 8 y 9 ).

\section{Discusión}

Los defectos óseos tibiales mediales son comúnmente encontrados durante la artroplastia total de rodilla. No se recomienda realizar el corte tibial al nivel del defecto medial, ya que con cortes $>1 \mathrm{~cm}$ por debajo de la línea articular, la calidad y cantidad de hueso esponjoso de soporte disminuye. De igual importancia son las inserciones de la banda iliotibial, la pes anserinus, el tendón rotuliano y el ligamento cruzado posterior, las cuales se pueden ver comprometidas con cortes más distales. ${ }^{8}$

Se han descrito varias opciones para el manejo de defectos periféricos tibiales no contenidos, incluyendo el relleno del defecto con cemento, ${ }^{9}$ el uso de tornillos y malla metálica, ${ }^{10}$ el uso de aumentos con cuñas de metal ${ }^{11} \mathrm{y}$ el uso de implantes con vástagos para fijación diafisaria. ${ }^{12}$

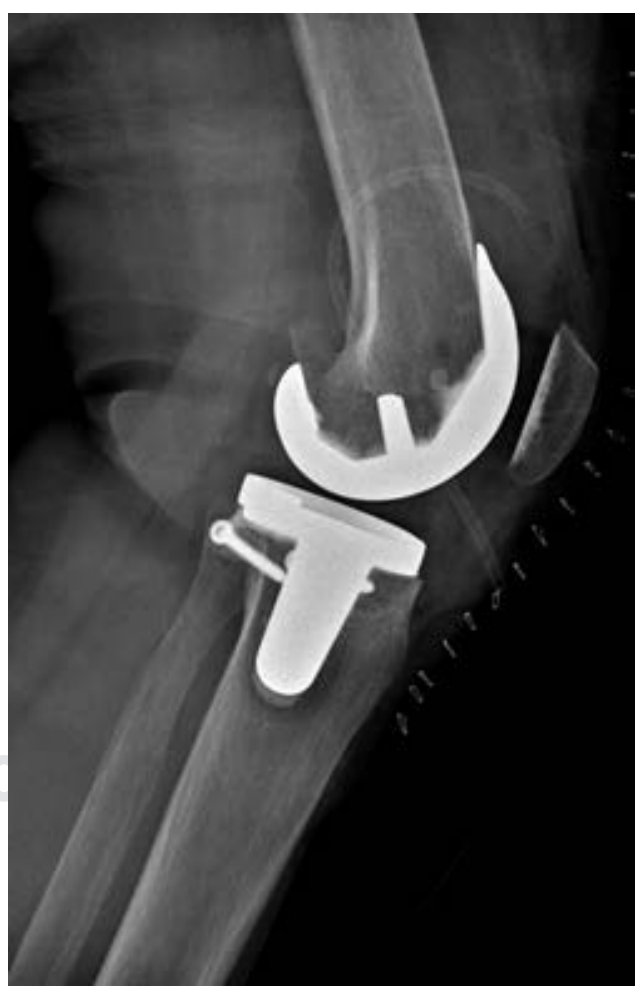

Figura 7: Radiografía lateral de rodilla izquierda en postquirúrgico inmediato. 


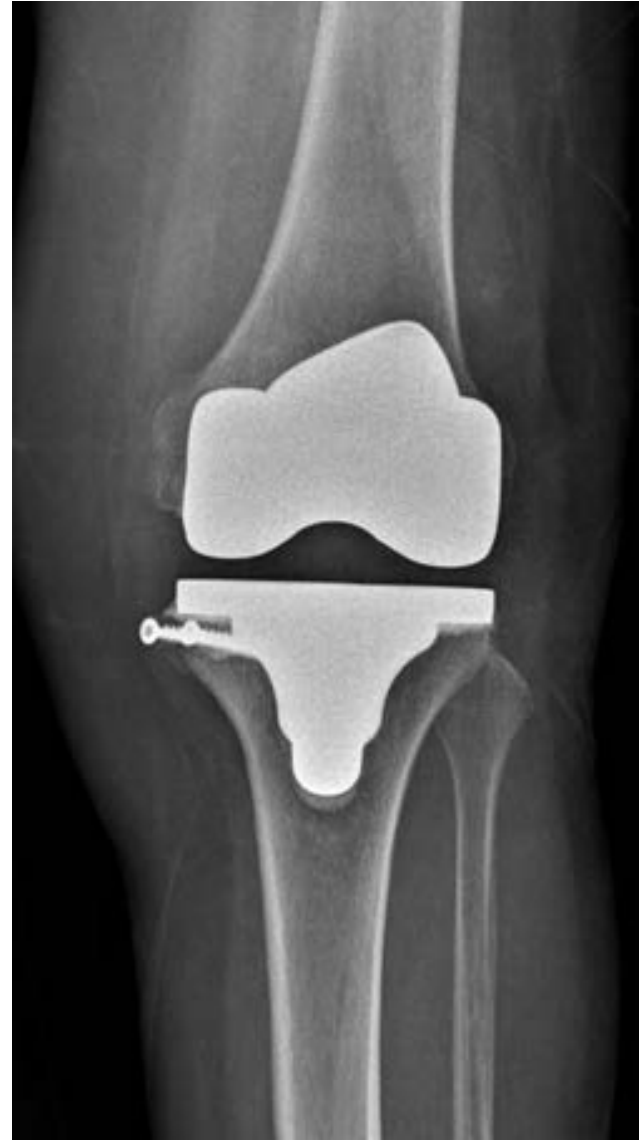

Figura 8: Radiografía anteroposterior de rodilla izquierda en bipedestación a las cuatro semanas de postquirúrgico.

En 1986, Windsor publicó su técnica para el tratamiento de defectos tibiales con injerto autólogo durante la artroplastia total de rodilla, obtenido durante los cortes femorales o tibiales necesarios para la colocación del implante. ${ }^{13}$

Debido a la disponibilidad y facilidad de colocación y fijación, el injerto autólogo estructural representa una opción viable y costeable para el tratamiento de defectos tibiales no contenidos.

En un estudio por Rai y colaboradores se realizó seguimiento de 80 pacientes sometidos a artroplastia total de rodilla con deformidad en varo promedio de $25^{\circ}\left(25-40^{\circ}\right)$ en ángulo femorotibial, a los cuales se les colocó injerto autólogo para el defecto tibial medial, con un seguimiento promedio de tres años (tres a seis años), encontrando consolidación e integración completa a los 4.5 meses en 73 de las 80 rodillas tratadas con puntajes de buenos a excelentes en escala de WOMAC y Knee
Society Score, reportando reabsorción de injerto en tres pacientes y pérdida del injerto por infección temprana en tres casos. ${ }^{14}$

Yoon y colaboradores publicaron una serie de 19 pacientes (22 rodillas) sometidos a artroplastia total de rodilla primaria con deformidad en varo, en los cuales se colocó autoinjerto estructural para defecto medial tibial, que fue fijado con clavillos Kirschner, encontrando mejoría significativa en el Knee Society Score, así como en rangos de movimiento pre- y postquirúrgicos. Se realizó seguimiento promedio de 30 meses, con integración completa en todos los casos, sin encontrar reabsorción de injerto, aflojamiento o migración de implante o clavillos Kirschner. ${ }^{15}$

En un estudio comparativo por Arslan y su equipo se estudió el resultado postquirúrgico entre casos que requirieron autoinjerto para defecto medial tibial en artroplastia total de rodilla primaria, contra pacientes que no requirieron autoinjerto para el mismo procedimiento, con un seguimiento mínimo de tres años, sin encontrar diferencias significativas entre grupos para el puntaje en escala de WOMAC, rangos

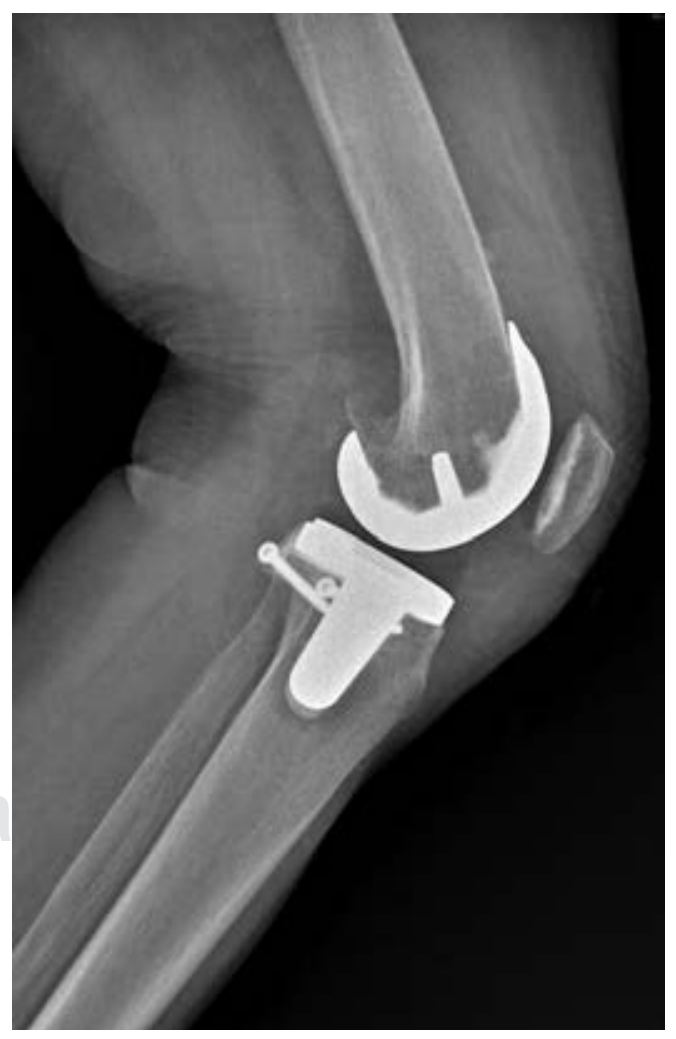

Figura 9: Radiografía lateral de rodilla izquierda a las cuatro semanas de postquirúrgico. 
de movimiento o angulación en eje mecánico, con resultados buenos a excelentes en ambos grupos, sin encontrar complicaciones relacionadas con la reconstrucción con injerto autólogo. ${ }^{16}$

Kharbanda y su grupo realizaron un análisis retrospectivo de 54 rodillas en 34 pacientes sometidos

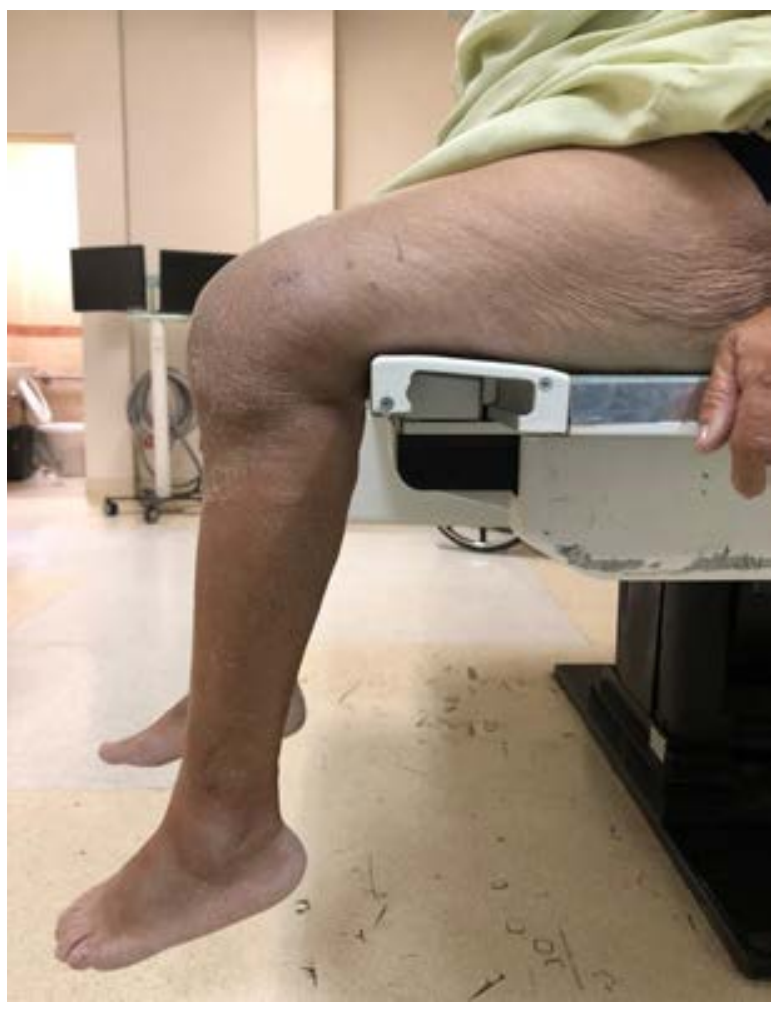

Figura 10: Flexión activa a las cuatro semanas de postquirúrgico.

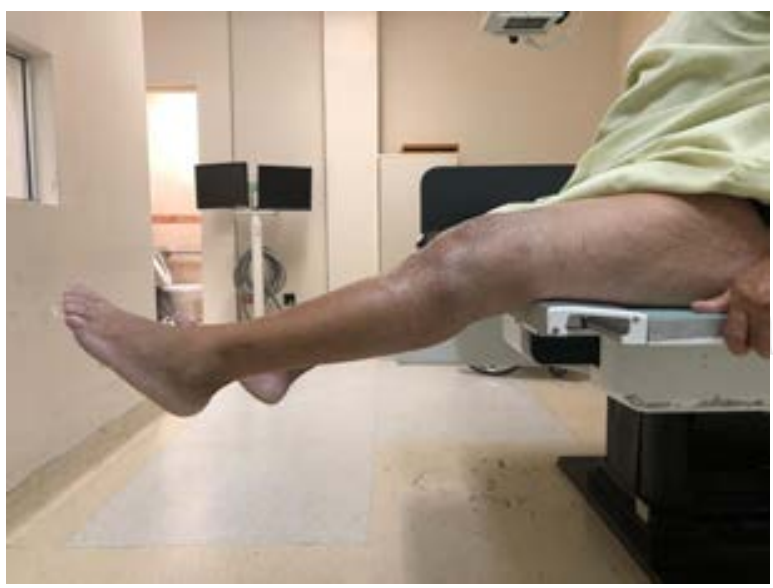

Figura 11: Extensión activa a las cuatro semanas de postquirúrgico.

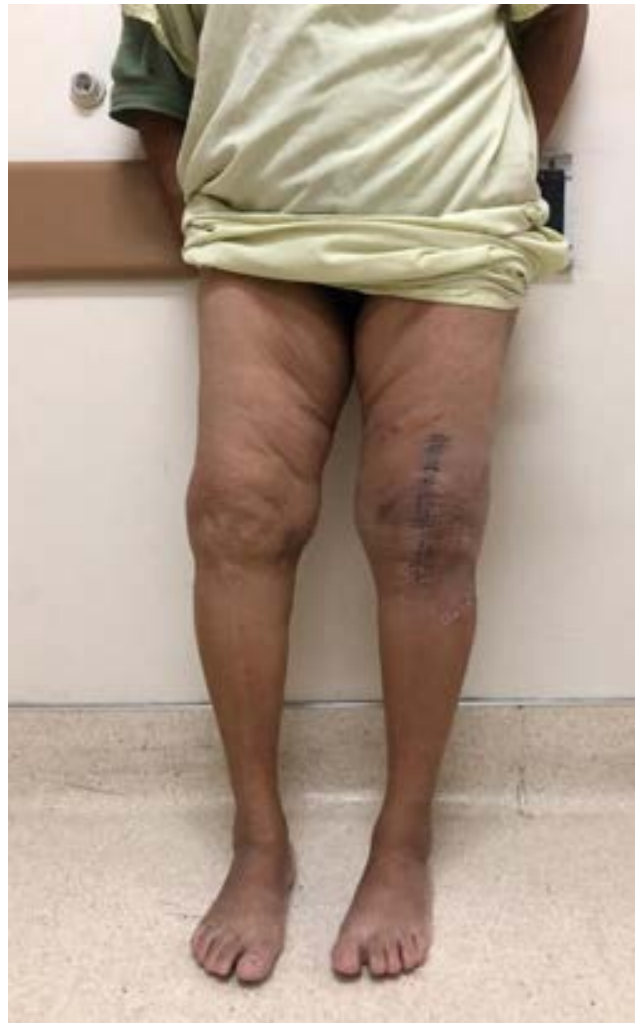

Figura 12: Corrección de genu varo a las cuatro semanas de postquirúrgico.

a artroplastia total de rodilla primaria con deformidad severa en varo que requirieron injerto autólogo para reconstrucción tibial por defectos $>5 \mathrm{~mm}$ posterior a corte convencional y angulación en varo promedio de $28^{\circ}$, con seguimiento promedio de 7.8 años, encontrando integración completa de injerto en todos los pacientes a los 4.5 meses en promedio, con resultados buenos a excelentes en escala de WOMAC y Knee Society Score, sin complicaciones tales como reabsorción de injerto, aflojamiento de componentes por efecto de batería (reacción electroquímica que se produce al realizarse contacto entre dos metales) 0 aflojamiento de tornillos. ${ }^{8}$

En el caso aquí reportado, se utilizó injerto autólogo proveniente del corte de chaflán anterior femoral para la reconstrucción tibial medial, el cual se fijó con tornillos de esponjosa de $4.0 \mathrm{~mm}$, sin encontrar datos de reabsorción, aflojamiento o fragmentación en un seguimiento temprano de ocho semanas, con mejoría significativa de sintomatología y aumento de funcionalidad (Figuras 10 a 12). 


\section{Conclusiones}

La utilización de injerto autólogo proveniente de corte femoral o tibial para la reconstrucción de platillo tibial en rodillas con deformidad severa en varo con defectos no contenidos es una opción segura, con amplia disponibilidad y fácil de realizar, que no representa una carga económica extra ni un aumento de morbilidad significativa para pacientes con este tipo de deformidad.

\section{Agradecimientos}

Al Hospital General de Mexicali y al Servicio de Ortopedia y Traumatología, así como a todo el cuerpo de maestros y compañeros residentes.

\section{Referencias}

1. Luthra JS, Habsi SAL. Biological reconstruction of varus deformity in primary total knee replacement using bone graft and description of a new technique. Journal of Orthopaedics, Trauma and Rehabilitation. 2018; 25: 69-72. doi: 10.1016/j. jotr.2017.11.001.

2. Hosaka K, Saito S, Oyama T, Fujimaki H, Cho E, Ishigaki K, et al. Union, knee alignment, and clinical outcomes of patients treated with autologous bone grafting for medial tibial defects in primary total knee arthroplasty. Orthopedics. 2017; 40 (4): e604-e608. doi: 10.3928/01477447-20170418-01.

3. Bauman RD, Lewallen DG, Hanssen AD. Limitations of structural allograft in revision total knee arthroplasty. Clin Orthop Relat Res. 2009; 467 (3): 818-824. doi: 10.1007/ s11999-008-0679-4.

4. Rand JA. Bone deficiency in total knee arthroplasty, use of metal wedge augmentation. Clin Orthop Relat Res. 1991; 271: 6371. https://journals.Iww.com/clinorthop/Abstract/1991/10000/ Use_of_Metal_Wedge_Augmentation.9.aspx.

5. Tanwar YS, Kharbanda Y, Bhargava H, Attri K, Bandil A. Midterm results of impaction bone grafting in tibial bone defects in complex primary knee arthroplasty for severe varus deformity. SICOT J. 2019; 5: 2. doi: 10.1051/sicotj/2018056.

6. Ahmed I, Logan M, Alipour F, Dashti H, Hadden WA. Autogenous bone grafting of uncontained bony defects of tibia during total knee arthroplasty. a 10-year follow up. J Arthroplasty. 2008; 23 (5): 744-750. doi: 10.1016/j. arth.2007.08.021.

7. Lagunas-Sánchez CE, Matehuala-García J, Jardines-Flores $H$, Mongragón-Rosete R. Artroplastia total de rodilla primaria en paciente con defecto óseo tibial posteromedial mayor de 5 cm: reporte de un caso. Ortho-tips. 2018; 14 (2): 93-97.

8. Kharbanda $\mathrm{Y}$, Sharma M. Autograft reconstructions for bone defects in primary total knee replacement in severe varus knees. Indian J Orthop. 2014; 48 (3): 313-318. doi: 10.4103/0019-5413.132525.

9. Hejna MJ, Gitelis S. Allograft prosthetic composite replacement for bone tumors. Published online. 1997, 18-24.

10. Ritter MA, Keating EM, Faris PM. Screw and cement fixation of large defects in total knee arthroplasty: a sequel. J Arthroplasty. 1993; 8 (1): 63-65. doi: 10.1016/S08835403(06)80109-9.

11. Chung KS, Lee JK, Lee HJ, Choi CH. Double metal tibial blocks augmentation in total knee arthroplasty. Knee Surg Sports Traumatol Arthrosc. 2016; 24 (1): 214-220. doi: 10.1007/ s00167-014-3368-8.

12. Kang SG, Park $\mathrm{CH}$, Song SJ. Stem fixation in revision total knee arthroplasty: indications, stem dimensions, and fixation methods. Knee Surg Relat Res. 2018; 30 (3): 187-192. doi: 10.5792/ksrr.18.019.

13. Windsor RE, Insall JN, Sculco TP. Bone grafting of tibial defects in primary and revision total knee arthroplasty. Clin Orthop Relat Res. 1986; 205: 132-137. doi: 10.1097/00003086198604000-00016.

14. Rai SK, Varma R, Wani SS. To study the outcome of autograft reconstructions for medial tibial bone defects in primary total knee replacement in 80 cases of severe varus knees. International Journal of Scientific Research. 2017; 6 (3): 99101. doi: $10.36106 /$ ijsr.

15. Yoon J, Seo I, Shin Y. Use of autogenous onlay bone graft for uncontained tibial bone defects in primary total knee arthroplasty. BMC Musculoskeletal Disorders 2017; 1-7. doi: 10.1186/s12891-017-1826-4.

16. Arslan A. Using tibia proximal cut autograft in advanced varus knee deformity in total knee arthroplasty; Outcomes Compared to the Abstract? Open Orthop. 2018; 12: 405-410. doi: $10.2174 / 1874325001812010405$.

\section{Conflicto de intereses}

No existe conflicto de intereses por parte de la institución ni de los médicos involucrados en el caso clínico. 